\title{
Cogitare
Enfermagem
}

\section{FIBRILAÇÃO ATRIAL EM PACIENTES SUBMETIDOS À HEMODIÁLISE CONTÍNUA}

\author{
Lucas da Piedade Primon, Fernando Riegel ${ }^{2}$, Diana da Silva Russo ${ }^{3}$
}

\section{RESUMO}

Objetivo: descrever a prevalência de fibrilação atrial, o perfil epidemiológico e o tratamento utilizado em pacientes submetidos à hemodiálise contínua.

Método: estudo quantitativo, transversal, realizado em 2017, com 110 pacientes internados num Centro de Terapia Intensiva de hospital privado de grande porte de Porto Alegre.

Resultados: evidenciaram-se 36 (32,7\%) ocorrências de fibrilação atrial durante a hemodiálise, com predominância da raça branca, sexo masculino em 25 (69,4\%) e idade média de 75,2 $\pm 10,5$. Verificou-se que $22(61,1 \%)$ desencadearam o primeiro episódio de Fibrilação Atrial. A hipertensão arterial sistêmica e a obesidade foram as comorbidades de maior prevalência. Houve evidência maior de cardioversão química em 30 e elétrica em seis pacientes.

Conclusão: evidenciou-se alta prevalência de fibrilação atrial em pacientes submetidos à hemodiálise contínua, o que denota a necessidade de competências específicas dos profissionais para a identificação, manejo das intercorrências cardiológicas e registros de qualidade acerca dos eventos.

DESCRITORES: Fibrilação Atrial; Arritmias cardíacas; Doenças Cardiovasculares; Diálise renal; Enfermagem.

COMO REFERENCIAR ESTE ARTIGO:

Primon $L$ da $P$, Riegel $F$, Russo $D$ da $S$. Fibrilação atrial em pacientes submetidos à hemodiálise contínua. Cogitare enferm. [Internet]. 2019 [acesso em "colocar data de acesso, dia, mês abreviado e ano"]; 24. Disponível em: http://dx.doi.org/10.5380/ce.v24i0.60386.

\section{(c) (1)}

Este obra está licenciado com uma Licença Creative Commons Atribuição 4.0 Internacional.

${ }^{1}$ Enfermeiro. Pós-graduando em Terapia Intensiva e Emergência. Enfermeiro Assistencial do Hospital Moinhos de Vento. Porto Alegre, RS, Brasil. 1

${ }^{2}$ Enfermeiro. Doutor em Enfermagem. Universidade Federal do Rio Grande do Sul. Porto Alegre, RS, Brasil. 9

${ }^{3}$ Enfermeira. Doutoranda do Programa de Ciências da Saúde da Universidade Federal do Rio Grande do Sul. Porto Alegre, RS, Brasil. 


\title{
ATRIAL FIBRILLATION IN PATIENTS RECEIVING CONTINUOUS HEMODIALYSIS
}

\begin{abstract}
Objective: to describe the prevalence of atrial fibrillation, the epidemiological profile and the treatment used in patients undergoing continuous hemodialysis.

Method: Quantitative cross-sectional study conducted in 2017 with 110 patients admitted to an intensive care center of a large private hospital in Porto Alegre.

Results: The study found 36 (32.7\%) episodes of atrial fibrillation during hemodialysis. Most patients were white males: 25 (69.4\%) with a mean age of $75.2 \pm 10$. Also, $22(61.1 \%)$ experienced their first episode of atrial fibrillation. Systemic arterial hypertension and obesity were the most prevalent comorbidities. There was evidence of chemical cardioversion in 30 patients and electrical cardioversion in six patients.

Conclusion: There was a high prevalence of atrial fibrillation in patients receiving continuous hemodialysis. Thus, health professionals must have specific skills for the identification and management of heart disorders and accurate and complete records about the events.
\end{abstract}

DESCRIPTORS: Atrial fibrillation; Heart arrhythmias; Cardiovascular diseases; Kidney dialysis; Nursing.

\section{FIBRILACIÓN AURICULAR EN PACIENTES SOMETIDOS A LA HEMODIÁLISIS CONTINUA}

\section{RESUMEN}

Objetivo: describir la prevalencia de fibrilación auricular, el perfil epidemiológico y el tratamiento que se utiliza en pacientes sometidos a la hemodiálisis constante.

Método: estudio cuantitativo, trasversal, que se realizó en 2017, con 110 pacientes ingresados en un Centro de Terapia Intensiva de un gran hospital particular en Porto Alegre.

Resultados: se evidenciaron 36 (32,7\%) ocurrencias de fibrilación auricular durante la hemodiálisis, con predominancia de la raza blanca, sexo masculino en $25(69,4 \%)$ y promedio de edad de 75,2 $\pm 10,5$. Se verificó que $22(61,1 \%)$ presentaron el primero episodio de Fibrilación Auricular. La hipertensión arterial sistémica y la obesidad fueron las comorbilidades de más prevalencia. Hubo evidencia mayor de cardioversión farmacológica en 30 y eléctrica en seis pacientes.

Conclusión: se evidenció alta prevalencia de fibrilación auricular en pacientes sometidos a la hemodiálisis constante, lo que apunta para la necesidad de competencias específicas de los profesionales para la identificación, la administración de las complicaciones cardiológicas y los registros de cualidad acerca de los eventos.

DESCRIPTORES: Fibrilación Auricular; Arritmias cardíacas; Enfermedades Cardiovasculares; Diálisis renal; Enfermería. 
No atual cenário de saúde brasileira e mundial, destacam-se as doenças cardiovasculares com elevação da sua incidência. Tal fato pode estar relacionado com o aumento da complexidade e de mudanças no perfil epidemiológico das doenças, causando maiores índices de internações e custos hospitalares.

Conforme dados da OMS (Organização Mundial de Saúde), em 2016, e do IBGE (Instituto Brasileiro de Geografia e Estatística), em 2013, cardiopatias ainda são as principais causas de morte no Brasil ${ }^{(1-2)}$. Em 2012, constatou-se que 17,5 milhões de pessoas morreram por doenças cardiovasculares, resultando em $31 \%$ de óbitos no nível global. Mais de $3 / 4$ desse valor estão representados por países com elevado número de pessoas com baixa e média renda. Além disso, essa população não possui rede de atenção integrada, nos diferentes níveis de atenção à saúde, capaz de atender todas as necessidades de saúde dos indivíduos ${ }^{(2)}$.

Dentre as arritmias cardíacas associadas às cardiopatias, pode-se destacar a Fibrilação Atrial (FA). Estima-se que esta seja responsável por um terço das internações hospitalares por arritmia ${ }^{(3)}$. Esse dado torna-se ainda mais alarmante quando é evidenciada a prevalência da doença, pois pode atingir mais de 4,8 milhões de brasileiros e mais de 2 milhões de americanos $^{(4)}$.

Verifica-se, nesse contexto, que a FA é considerada um importante problema de saúde, pois causa aumento significativo do consumo de recursos públicos em razão do crescimento considerável na população brasileira(5). Mesmo sendo maior a prevalência ajustada por idade em homens, acima de 75 anos, a prevalência em mulheres chega a $60 \%(3)$. Países desenvolvidos relatam crescimento na incidência e prevalência de FA, relacionada à expectativa de vida e ao aumento da população idosa. Estima-se que, nas próximas décadas, o crescimento será maior que o dobro ${ }^{(3,6-7)}$.

FA é a arritmia supraventricular mais comum na prática clínica, ocasionada por uma desordem eletrofisiológica na comunicação entre o nó sinoatrial e os átrios, com focos ectópicos atriais que tentam conduzir o ritmo cardíaco, levando-os à frequência dos batimentos cardíacos irregulares em torno de $350 \mathrm{bpm}$. Deste modo, os átrios perdem a função de contração e passam a "fibrilar"(6,8-11). Os registros eletrocardiográficos evidenciam ondas $f$ (fibrilatórias) no traçado, variando sua forma e também sua amplitude ${ }^{(9)}$.

Cerca de $30 \%$ dos pacientes são assintomáticos, porém, nesse caso, podem apresentar as seguintes manifestações clínicas: palpitações, síncope, complicações embólicas, poliúria, pulso irregular e cansaço ${ }^{(3,5)}$. As alterações são confirmadas precisamente após o eletrocardiograma (ECG), mas outros exames servem para auxiliar na investigação da FA, como por exemplo: o uso de Holter 24h, ecocardiograma, radiografia de tórax e exames laboratoriais ${ }^{(3)}$.

Estudos demonstram a influência de fatores considerados não cardiovasculares como a idade e o sexo, o diabetes (DM), o hipertireoidismo, o uso de bebidas alcóolicas, a obesidade, o histórico familiar, o excesso de atividade física; e fatores genéticos, tendo predisposição significativa de morte para pacientes com presença de insuficiência renal (IR), câncer, doença pulmonar obstrutiva (DPOC), síndrome de apneia obstrutiva do sono e sepse ${ }^{(5,7)}$.

O tratamento de FA pode ser clínico ou cirúrgico. No que tange às possibilidades de tratamento clínico, a escolha inicial será a cardioversão química através do uso de antiarrítmicos para haver reversão espontânea ${ }^{(5)}$. Outra opção é a cardioversão elétrica realizada com o uso do desfibrilador ${ }^{(3,5)}$. Em relação ao tratamento cirúrgico, a principal indicação é a ablação ou crioablação por cateter com a intenção de inativar estas células ${ }^{(3)}$.

O uso de anticoagulantes será um dos primeiros medicamentos a serem indicados no tratamento como prevenção ao risco de formação de trombos, principalmente se estiver 
apresentando a arritmia por $\geq 48 \mathrm{~h}$ ou por tempo indeterminado ${ }^{(3-5)}$. $O$ risco da FA evoluir para um acidente vascular encefálico isquêmico (AVE) aumenta em 5 vezes mais, pelo fato do átrio não contrair de forma efetiva, estagnando o sangue na cavidade atrial e favorecendo a formação de coágulos ${ }^{(4,10)}$.

Doença cardiovascular (DCV), associada à doença renal crônica (DRC), aumenta o risco de óbitos em pacientes dialíticos, cuja mortalidade chega a ser 10 a 20 vezes maior se comparada à população em geral. Faz-se importante destacar que $30 \%$ a $44 \%$ dos pacientes em tratamento com hemodiálise pela primeira vez apresentam $\mathrm{DCV}^{(12)}$.

A hemodiálise é utilizada para filtrar o sangue dos indivíduos através de uma membrana semipermeável. O sangue passa no interior de um filtro no sentido contrário à solução dialítica em sistema contracorrente e por fora das fibras do capilar, proporcionando trocas osmóticas. A hemodiálise contínua, por sua vez, é utilizada geralmente em pacientes hemodinamicamente instáveis que necessitam de um controle maior da uremia, equilíbrio acidobásico e remoção de líquidos. Essa técnica é realizada em unidades de terapia intensiva, tendo duração de $24 \mathrm{~h}$ em média, mas podendo durar alguns dias sem interrupção. Os fluxos de sangue e dialisado são baixos, proporcionando, dessa forma, tratamento de alta eficácia e com menor dano hemodinâmico(13-14).

Pacientes com DRC submetidos à terapia dialítica apresentam alta prevalência de anormalidades no traçado eletrocardiográfico, sendo FA a arritmia mais comum nesses pacientes em hemodiálise, por inúmeros fatores, mas também por baixos níveis séricos de albumina ${ }^{(15)}$. No entanto, observa-se a relação de FA com a insuficiência renal, necessitando de tratamento dialítico ou não. Nesse contexto, constatou-se que o paciente com tais comorbidades relacionadas tem o risco aumentado em evoluir para AVE isquêmico, infarto do miocárdio e tromboembolismo(16).

Frente ao exposto, o objetivo deste estudo foi descrever a prevalência de fibrilação atrial, o perfil epidemiológico e o tratamento utilizado em pacientes internados, em centro de tratamento intensivo, submetidos à hemodiálise contínua.

\section{MÉTODO}

Trata-se de um estudo com delineamento quantitativo do tipo transversal(17-19), desenvolvido com pacientes que estiveram internados em um hospital privado de grandé porte na cidade de Porto Alegre, Rio Grande do Sul, Brasil.

A amostra foi constituída por 110 pacientes, internados no Centro de Tratamento Intensivo, composto por 31 leitos e dividido em 3 áreas. Foram incluídos, no estudo, participantes com idade igual ou superior a 18 anos e sem estabelecer limite de idade máxima, de ambos os sexos e de qualquer etnia, que realizaram hemodiálise contínua, podendo haver diagnóstico de fibrilação atrial prévio e/ou já ter realizado tratamento com hemodiálise convencional durante a mesma internação. Foram excluídos dados dos sujeitos que não apresentaram informações completas e claras no prontuário.

Foram incluídos os pacientes internados no período de 01/01/2015 a 31/12/2016. Optou-se por este período, tendo em vista o recorte temporal exato de dois últimos anos que antecedessem a realização da pesquisa. Os fatores de riscos pesquisados no estudo foram: insuficiência cardíaca, doença valvar, hipertensão arterial sistêmica, cardiopatia isquêmica, sepse, acidente vascular encefálico, doença pulmonar obstrutiva crônica, apneia do sono, câncer, diabetes melittus, hipertireoidismo, obesidade e etilismo.

A coleta de dados ocorreu no período de 28 de setembro a 06 de outubro de 2017. Foi utilizado um instrumento elaborado pelos pesquisadores com as seguintes informações: registro do paciente, idade, etnia, gênero, registro de FA prévia, FA durante e após a hemodiálise, comorbidades prévias, complicações durante a internação e o tipo de anticoagulação. 
As variáveis quantitativas foram descritas por média e desvio padrão e as categóricas por frequências absolutas e relativas. Para avaliar a associação entre as variáveis, os testes qui-quadrado de Pearson ou exato de Fisher foram utilizados. Para complementar essas análises, o teste dos resíduos ajustados foi aplicado. O nível de significância adotado foi de $5 \%(p \leq 0,05)$. A análise estatística foi realizada utilizando o programa SPSS V.21.0.

A coleta de dados foi iniciada após a aprovação do projeto pelo Comitê de Ética em Pesquisa sob Protocolo de aprovação n².301.

\section{RESULTADOS}

Foram avaliados os prontuários de 110 pacientes em hemodiálise contínua internados em um Centro de Terapia Intensiva (CTI) entre nos anos de 2015 e 2016, representando 65 (59,1\%) pacientes internados no ano de 2015; e 45 (40,9\%) em 2016.

Dos 110 participantes do estudo, evidenciou-se que $29(26,4 \%)$ possuíam previamente o diagnóstico de fibrilação atrial; destes, 14 (48,3\%) participantes fibrilados também apresentaram episódios de FA em algum momento durante a terapia hemodialítica.

Para a apresentação da análise dos dados coletados, subdividiram-se os achados em categorias, sendo elas: caracterização da amostra e perfil epidemiológico, prevalência de Fibrilação Atrial (FA), comorbidades e complicações relacionadas à FA, métodos de tratamentos utilizados nos episódios de FA e métodos de anticoagulação utilizados na hemodiálise contínua. Na Tabela 1, apresentam-se os dados referentes à caracterização da amostra.

Tabela 1 - Caracterização da amostra. Porto Alegre, RS, Brasil, 2017

\begin{tabular}{|c|c|}
\hline Variáveis & $n=110(n \%)$ \\
\hline Idade (anos) - média \pm DP [min-máx.] & $74,8 \pm 14,2$ [23-99] \\
\hline \multicolumn{2}{|l|}{ Faixa etária } \\
\hline$<60$ & $15(13,6)$ \\
\hline $60-69$ & $18(16,4)$ \\
\hline $70-79$ & $24(21,8)$ \\
\hline 80 ou mais & $53(48,2)$ \\
\hline \multicolumn{2}{|l|}{ Sexo } \\
\hline Masculino & $70(63,6)$ \\
\hline Feminino & $40(36,4)$ \\
\hline \multicolumn{2}{|l|}{ Cor } \\
\hline Branca & $109(99,1)$ \\
\hline Amarela & $1(0,9)$ \\
\hline Preta & 0 \\
\hline
\end{tabular}

Das comorbidades prévias apresentadas na amostra geral dos pacientes selecionados, destacaram-se: Hipertensão Arterial Sistêmica em 73 pacientes $(66,4 \%)$, Câncer em 39 (35,5\%), Diabetes Mellitus em 34 (30,9\%), Cardiopatia Isquêmica em 30 (27,3\%), Insuficiência 
Renal Crônica em 29 (26,4\%), Insuficiência Cardíaca em 18 (16,4\%), Doença Pulmonar Obstrutiva Crônica em 16 (14,5), Acidente Vascular Encefálico em 16 (14,5\%), sendo 15 do tipo isquêmico; $13(11,8 \%)$ dos participante das pesquisas eram pós-operatório de cirurgia de revascularização do miocárdio (CRM), Vasculopatia Periférica com oito (7,3\%) e Insuficiência Cardíaca Valvar com seis (5,5\%).

No que se refere aos fatores de risco de maior predominância, houve destaque para o Tabagismo, sendo ativo ou ex-fumante, com 27 participantes (24,5\%); o Etilismo com seis $(5,5 \%)$ e a Obesidade em cinco $(4,5 \%)$ dos pacientes incluídos neste estudo.

Nove $(8,2 \%)$ participantes apresentaram alguma arritmia, porém não foi esclarecido em prontuário qual o tipo identificado, e por fim, nenhum dos pacientes apresentou apneia do sono, e nem hipertireoidismo, somente hipotireoidismo $18(16,4 \%)$. Na Figura 1 , apresenta-se a prevalência das comorbidades evidenciadas na amostra $(n=110)$.

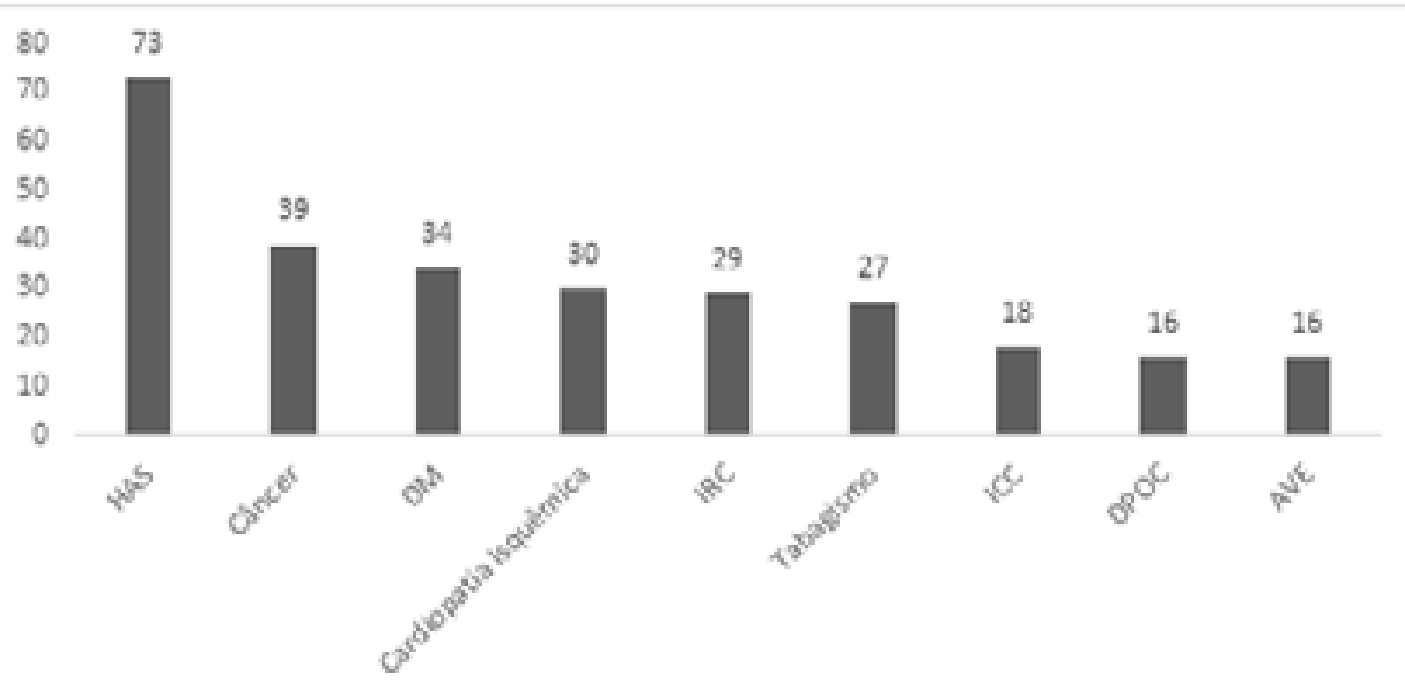

Figura 1 - Prevalência de comorbidades ( $n=110)$. Porto Alegre, RS, Brasil, 2017

Evidenciou-se que $29(26,4 \%)$ possuíam previamente o diagnóstico de fibrilação atrial; destes, 14 (48,3\%) participantes fibrilados também apresentaram episódios de FA em algum momento durante a terapia hemodialítica.

Em relação aos 36 (32,7\%) participantes que fibrilaram durante a hemodiálise contínua em sua totalidade, quando avaliados, verificou-se que $22(61,1 \%)$ desencadearam o primeiro episódio de FA. Entre os pacientes que fibrilaram após o término da hemodiálise, o resultado foi de $13(11,8 \%)$ da amostra, tendo em vista que nove $(69,2 \%)$ desta amostra também fibrilaram durante a sessão de hemodiálise, e oito $(61,5 \%)$ eram fibrilados prévios. Apenas um $(7,7 \%)$ participante apresentou somente o primeiro episódio de fibrilação atrial após o término da hemodiálise contínua (Figura 2). 


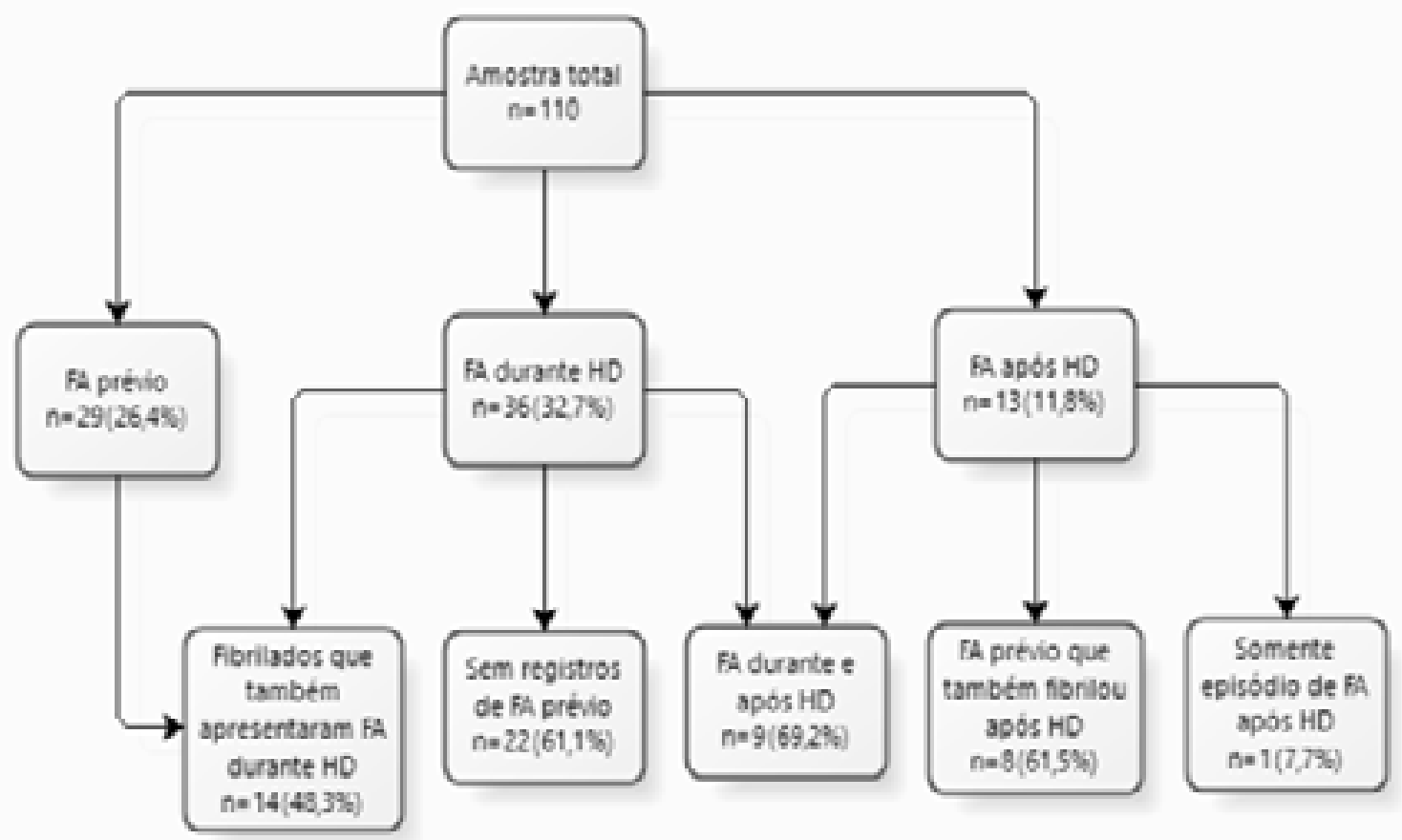

Figura 2 - Prevalência de Fibrilação Atrial (FA). Porto Alegre, RS, Brasil, 2017

As comorbidades e as complicações relacionadas à fibrilação atrial foram obtidas com base no número de participantes que fibrilaram durante hemodiálise (HD), ou seja, 36 (32,7\%); em seguida, comparou-se esse número ao número de participantes que não apresentaram episódios de FA durante a HD, sendo $74(67,3 \%)$.

Associando-se os participantes que apresentaram FA durante HD contínua 36 (32,7\%) com os que não tiveram episódios de FA, 74 (67,3\%) obtiveram-se os seguintes resultados: o sexo masculino apresentou maior prevalência de FA em HD contínua, com $25(69,4 \%)$ casos; a média de idade foi de 75,2 $\pm 10,5$ anos; no que se refere às participantes do sexo feminino, houve $11(30,6 \%)$ casos e a média de idade foi de 80,5 9,6 anos.

Dentre as comorbidades pertinentes ao estudo, a hipertensão arterial sistêmica (HAS) destacou-se estatisticamente, mostrando-se significativa e estando presente entre os participantes que fibrilaram em HD contínua, com $30(83,3 \%)$ casos; já 43 (58,1\%) dos participantes não apresentaram FA, $p=0,016$.

A predominância de HAS foi evidenciada no sexo feminino, sendo 10 (90,9\%); já $20(80 \%)$ dos participantes eram do sexo masculino. Em relação aos fatores de risco, a obesidade em quatro $(11,1 \%)$ casos apresentou significância estatística, $p=0,039$. Outras comorbidades como cardiopatia isquêmica, IC, DPOC, AVE, insuficiência valvar e câncer não apresentaram significância neste estudo.

Outras complicações foram menos recorrentes, tais como: trombose venosa profunda (TVP) em dois $(9,1 \%)$ casos e infarto agudo do miocárdio (IAM) em um $(4,5 \%)$ caso.

Comparando-se com a amostra dos participantes que não fibrilaram durante HD contínua, 52 (70,3\%) evoluíram para óbito e 44 (59,5\%) tiveram sepse como complicação mais recorrente, não havendo significância estatística quando comparado aos participantes que fibrilaram, $p=0,314$.

Apresenta-se a seguir, a Figura 3, referente às comorbidades relacionadas aos participantes com FA durante a HD contínua diferenciando dos participantes sem episódios de FA. 


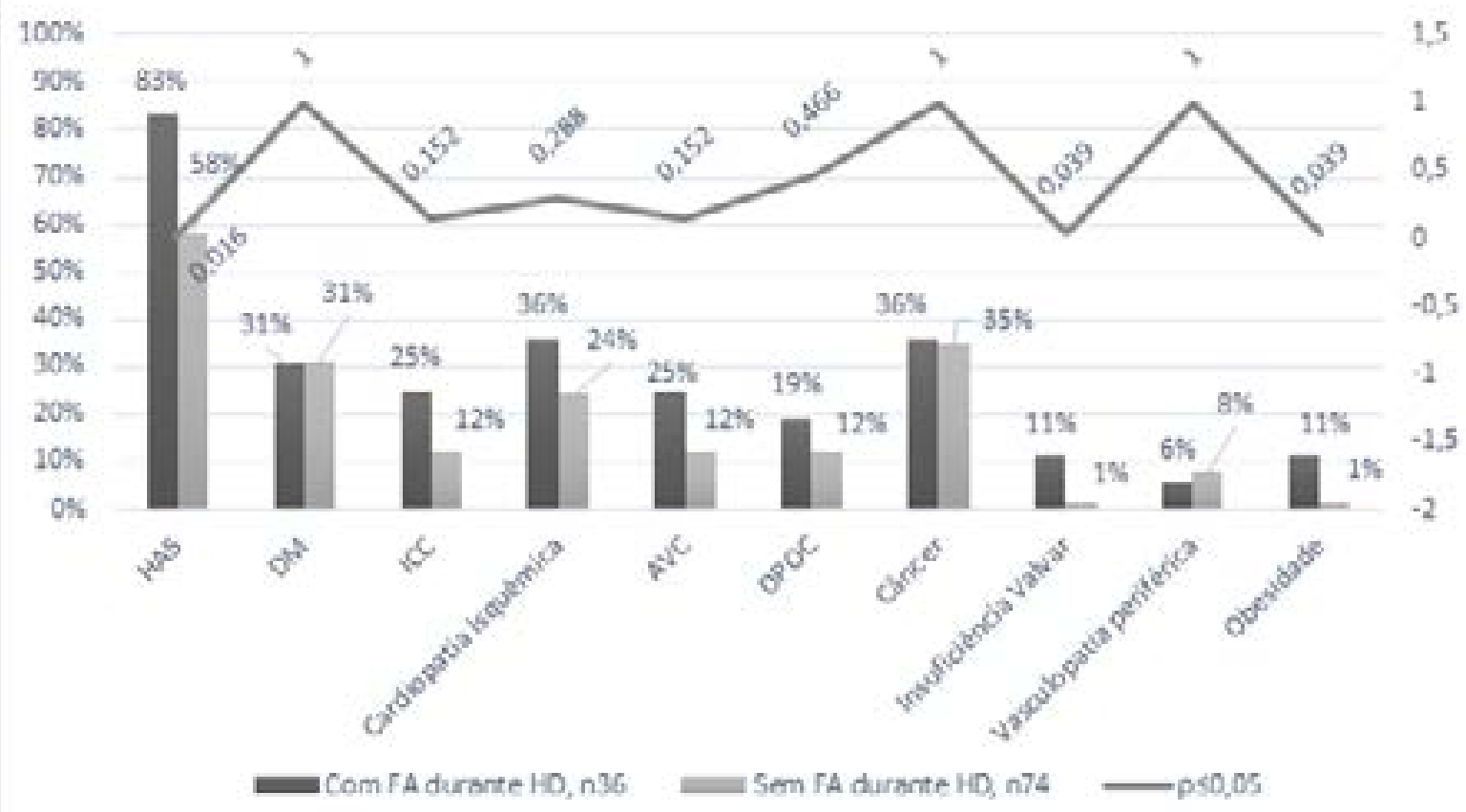

Figura 3 - Fibrilação atrial durante hemodiálise "versus" sem episódios de fibrilação. Porto Alegre, RS, Brasil, 2017

Com relação à abordagem terapêutica, foi possível evidenciar o uso da cardioversão elétrica, química e o uso concomitante de ambos os métodos de tratamento. Houve maior cardioversão química em 30 (83,3\%) dos casos de FA. Os medicamentos administrados foram: Amiodarona com apresentação de $(50 \mathrm{mg} / \mathrm{ml}) 3 \mathrm{ml}$ em 27 (90\%), Deslanosídeo $(0,2 \mathrm{mg} / \mathrm{ml}) 2 \mathrm{ml}$ em $13(43,3)$; e Tartarato de Metoprolol $(1 \mathrm{mg} / \mathrm{ml}) 5 \mathrm{ml}$ em $11(36,7)$, todos em bolus e por via intravenosa.

Em algumas ocorrências de FA, foram administradas mais de uma opção dos medicamentos citados acima; porém, foi evidente a preferência por amiodarona. A dosagem da amiodarona, no momento da FA, foi de duas ampolas de $3 \mathrm{ml}(50 \mathrm{mg} / \mathrm{ml})$ diluídas em $100 \mathrm{ml}$ de Soro glicosado 5\%, administrado em trinta minutos; e, para a manutenção nos casos intermitentes, foram identificadas como padrão da instituição, seis ampolas de $3 \mathrm{ml}(50 \mathrm{mg} / \mathrm{ml})$ diluídas em $232 \mathrm{ml}$ de soro glicosado $5 \%$, em um fluxo contínuo conforme orientação médica.

Quanto aos participantes que foram submetidos à cardioversão elétrica, o número se apresentou em seis $(16,7 \%)$. Todos os pacientes da amostra também foram medicados com antiarrítmicos antes e, não revertendo, foram induzidos a choques sincronizados; porém, os registros em prontuários eletrônicos não foram detalhados quanto à dosagem dos joules e se houve repetições das descargas.

Todos os pacientes estavam monitorados com monitor multiparamétrico da marca Dräger ${ }^{\circledR}$ e os que realizaram cardioversão elétrica também estavam monitorados com o monitor do carro de PCR (parada cardiorrespiratória).

Dos métodos de anticoagulação utilizados pela instituição para os pacientes incluídos na amostra, foi possível evidenciar a heparina 5.000 UI "Hepamax ${ }^{\circledR}$ " e a solução de citrato de sódio $4 \% 3000 \mathrm{ml}$. Dos 110 pacientes analisados, houve predominância do uso do anticoagulante citrato de sódio 4\% em 100 (90,9\%) participantes na terapia hemodialítica.

$\mathrm{Na}$ instituição estudada, a diluição da heparina é composta por $1 \mathrm{ml}$ de heparina para $19 \mathrm{ml}$ de soro fisiológico $0,9 \%$, adaptados à máquina de hemodiálise contínua (Prismaflex®), em um fluxo prescrito pelo médico nefrologista. 
Tratando-se do desfecho, $29(80,6 \%)$ dos participantes que fibrilaram durante a hemodiálise contínua evoluíram para óbito e sete $(19,4 \%)$ tiveram alta para a unidade de internação.

\section{DISCUSSÃO}

Comparando-se com a pesquisa ${ }^{(14)}$ e outra realizada no estado do Paraná que avaliou 168 participantes em hemodiálise convencional e destes 4,2\% apresentaram FA durante a sessão de hemodiálise, o presente estudo teve evidência de FA superior e a justificativa pode estar relacionada ao tipo de terapia hemodialítica utilizada e à necessidade do tratamento com ênfase à redução das alterações fisiológicas ${ }^{(8)}$.

Os dados referentes ao presente estudo evidenciaram a idade avançada sendo um fator predisponente para o surgimento da FA, e a maior prevalência de FA em sexo masculino, corroborando com os achados na literatura científica ${ }^{(3,6-8,20)}$.

Dos pacientes que apresentaram FA durante a HD no CTI adulto, $17(47,2 \%)$ evoluíram com sepse e/ou choque séptico. Em estudo realizado em Portugal, observouse a frequência de aparecimento de FA em pacientes com sepse, apesar das inúmeras alterações fisiológicas que ocorrem, pode-se citar a resposta inflamatória sistêmica e um aumento dos níveis de proteína $C$ reativa ${ }^{(7)}$, o que ficou evidente no estudo realizado em Porto Alegre.

Um estudo de coorte realizado em um hospital de São Paulo avaliou 3010 pacientes após serem submetidos à cirurgia de revascularização do miocárdio. Estes pacientes apresentaram semelhança no perfil epidemiológico, ou seja, 82,2\% eram hipertensos e $69,9 \%$ do sexo masculino, sendo que desses $36,6 \%$ possuíam DM. A complicação mais comum entre eles foram as arritmias cardíacas com 18,7\%, dentre elas $14,3 \%$ foram identificadas como FA e 4,4\% apresentaram lesão renal que necessitaram hemodiálise, apontando importante relação com a pesquisa realizada em Porto Alegre ${ }^{(21)}$.

O estudo evidenciou 70 (63,6\%) dos participantes do sexo masculino com hipertensão arterial, corroborando com os achados do estudo realizado em São Paulo que avaliou mais de três mil pacientes após a cirurgia cardíaca de revascularização o miocárdio. Em síntese, os achados da pesquisa estão de acordo com o estudo analisado confirmando a hipertensão como comorbidade mais frequente, a idade avançada, bem como o sexo masculino de maior prevalência de FA.

O medicamento amiodarona, de ação antiarrítmica, destaca-se na escolha primária em consonância com estudo publicado em 2015 pela Sociedade Brasileira de Cardiologia, o qual evidenciou a escolha notória da amiodarona em episódios isolados de FA antes e também após procedimentos de ablação, como prevenção de arritmias, sendo também utilizados como manutenção aos pacientes fibrilados crônicos em pós-alta hospitalar; entretanto, na apresentação em comprimidos, conforme prescrição médica ${ }^{(22)}$. A II Diretriz Brasileira de Fibrilação Atrial comprova a eficácia de amiodarona, sendo uma das primeiras alternativas de tratamento com antiarrítmicos ${ }^{(5)}$.

Estudo realizado em Cuba, abordando a cardioversão elétrica como modalidade terapêutica em FA, trouxe êxito no que tange à utilização da cardioversão elétrica em $81,2 \%$ dos casos. Ó autor também menciona a administração antecipada de amiodarona como coadjuvante ao tratamento de $F A$, pois a amiodarona auxilia fisiologicamente para que o choque tenha maior êxito, indo ao encontro dos achados deste estudo ${ }^{(23)}$.

A escolha do anticoagulante utilizado na terapia hemodialítica tem uma visão mais ampliada: a heparina tem ação sistêmica, podendo desencadear eventos hemorrágicos; por outro lado, o citrato de sódio $4 \%$ é utilizado por sua ação ter efeito apenas ao sistema de diálise, evitando a coagulação precoce do filtro capilar ${ }^{\{21)}$. 
O tratamento deve ser estudado a cada caso, uma vez que estes fatores são predisponentes para a instalação de um AVE, pois o paciente fibrilado necessita de um complemento com anticoagulantes, podendo ocasionar outras complicações tais como o acidente vascular encefálico. Já em casos de sepse, a FA também aumenta o tempo de internação hospitalar ${ }^{(7)}$.

Em estudo desenvolvido num hospital de São Paulo, avaliou-se a aplicabilidade do citrato de sódio 4\% no sistema de HD contínua. Em todos os parâmetros avaliados, 0 citrato teve êxito, no que tange à anticoagulação do sistema; nenhum paciente apresentou problemas com coágulos durante a hemodiálise ${ }^{(24)}$.

Os participantes da amostra que fizeram uso de heparina, seis $(5,5 \%)$, e até mesmo os que intercalaram entre heparina e citrato de sódio $4 \%$, quatro $(3,6 \%)$, no sistema de hemodiálise, não apresentaram nenhuma complicação relacionada a eventos hemorrágicos. Esses métodos de anticoagulação utilizados não apresentaram relação significativa com pacientes fibrilados.

O estudo apresentou como limitação a ausência de informações nos registros dos prontuários analisados, o que dificultou a coleta e análise dos dados.

\section{CONCLUSÃO}

Este estudo evidenciou uma prevalência alta de participantes que apresentaram fibrilação atrial durante a hemodiálise contínua. A hipertensão arterial sistêmica foi a comorbidade estatisticamente comprovada como significante nestes participantes em hemodiálise, como também a obesidade sendo fator extrínseco de maior recorrência. Com relação ao tratamento da FA, o estudo identificou que, no campo de estudo, aplicamse as Diretrizes Brasileiras de Fibrilação Atrial, quando foi observada a administração de amiodarona como tratamento imediato e de longo prazo em episódios de fibrilação.

Como desafio, este estudo trouxe para reflexão a importância dos registros padronizados no prontuário dos pacientes. Informações incompletas e uso de siglas, que dificultaram o entendimento das informações registradas, são razões para sugestão da utilização de Folha de Controle Assistencial em HD contínua.

Como sugestão para novos estudos está a utilização de resultados de exames laboratoriais, tais como: análise sérica de potássio, sódio, magnésio e cálcio, albumina, INR (Razão Normalizada Internacional), TP (Tempo de Protrombina) e KTTP (Tempo de Tromboplastina Parcial Ativado) para complementar a análise dos dados.

Ressalta-se a necessidade de estudos com ênfase nos cuidados de enfermagem e monitorização do paciente com FA em HD, que contribuam para qualificar as equipes multiprofissionais em cuidados intensivos, na identificação e tratamento precoce da FA, evitando, desse modo, prolongamento do tempo de internação, lesões cardíacas e complicações tromboembólicas com pacientes internados nos centros de terapia intensiva.

Os resultados dessa pesquisa apontam para a relevante atuação do enfermeiro no que se refere à realização da hemodiálise contínua, bem como na identificação de complicações relacionadas a esta terapia como é o caso da fibrilação atrial. Além disso, destacam-se as importantes funções de organização do trabalho, assistência direta aos pacientes, identificação dos sinais de arritmias cardíacas e capacitação das equipes, com vistas à atenção aos episódios de arritmias, e desta forma contribuir para o diagnóstico, monitorização e tratamento, tendo em vista que eventos como a fibrilação atrial podem ocasionar complicações e sequelas permanentes para pacientes submetidos à hemodiálise contínua. 
1. Instituto Brasileiro de Geografia e Estatística (IBGE). Estimativas de População 2017. [Internet]. 2017 [acesso em 04 dez 2017]. Disponível em: https://www.ibge.gov.br/estatisticas-novoportal/sociais/ populacao/9103-estimativas-de-populacao.html?\&t=resultados.

2. Organização Mundial da saúde (OMS). Doenças cardiovasculares [Internet]. Brasília: OMS; 2016 [acesso em 04 dez 2017]. Disponível em: http://www.paho.org/bra/index.php?option=com content\&view=article \&id=5253:doencas-cardiovasculares-\&catid=845: notiias \&ltemid=839.

3. Silva RMFL. Arritmias cardíacas. In: Pires MTB, Starling SV, organizadores. Manual de urgência em pronto-socorro. 10. ed. Rio de Janeiro: Guanabara Koogan; 2014. p. 50-60.

4. Silva GS, Martins SO, Cabral NL, Pontes Neto OM, Freitas GR, Oliveira Filho J, et al. Sociedade brasileira de doenças cerebrovasculares [Internet]. 2015 [acesso em 25 nov 2017]. Disponível em: http:// www.sbdcv.org.br/.

5. Magalhães LP, Figueiredo MJO, Cintia FD, SAAD EB, Kuniyoshi RR, Teixeira RA, et al. Sociedade brasileira de cardiologia. II Diretrizes brasileiras de fibrilação atrial. Arq. Bras. Cardiol. [Internet]. 2016 [acesso 25 nov 2017]; 106(4). Disponível em: http://www.scielo.br/pdf/abc/v106n4s2/0066-782X-abc-10604-s2-0001.pdf.

6. Renoux C, Patenaude V, Suissa S. Incidence, mortality, and sex diferences of non-valvular atrial fibrillation: a population-based study. J. Am. Heart Assoc. [Internet] 2014 [acesso em 25 nov 2017]; 3(6). Disponível em: http://dx.doi.org/10.1161/JAHA.114.001402.

7. Ferreira C, Providência R, Ferreira MJ, Gonçalves LM. Fibrilação atrial e doenças não cardiovasculares: uma revisão sistemática. Arq. Bras. Cardiol. [Internet] 2015 [acesso em 25 nov 2017]; 105(5). Disponível em: http://dx.doi.org/10.5935/abc.20150142.

8. Peres LAB, Luiz AA, Espiga MC, Spivakoski CS. Fibrilação atrial em pacientes crônicos em hemodiálise. Rev. Soc. Bras. Clín. Med. [Internet]. 2012 [acesso em 25 nov 2017]; 10(6). Disponível em: http://files.bvs. br/upload/S/1679-1010/2012/v10n6/a3179.pdf.

9. Folla CO, Melo CCS, Silva RCG e. Fatores preditivos de fibrilação atrial no pós-operatório de revascularização do miocárdio. Einstein [Internet]. 2016 [acesso em 25 nov 2017];14(4). Disponível em: http://dx.doi.org/10.1590/s1679-45082016ao3673.

10. Justo FA, Silva AFG. Aspectos epidemiológicos da fibrilação atrial. Rev. Med. [Internet]. 2014 [acesso em 25 nov 2017]; 93(1). Disponível em: https://doi.org/10.11606/issn.1679-9836.v93i1p1-13.

11. Brick AV, Braile DM. Surgical ablation of atrial fibrillation using energy sources. Rev. Bras Cir. Cadiovasc. [Internet]. 2015 [acesso em 25 nov 2017]; 30(6). Disponível em: http://dx.doi. org/10.5935/1678-9741.20150078.

12. Burmeister JE, Mosmann CB, Costa VB, Saraiva RT, Grandi RR, Bastos JP, et al. Prevalence of Cardiovascular Risk Factors in Hemodialysis Patients - The CORDIAL Study. Arq. Bras. Cardiol. [Internet]. 2014 [acesso em 25 nov 2017]; 102(5). Disponível em: http://dx.doi.org/10.5935/abc.20140048.

13. Mendes NT, Amorim CP. Sistema renal - principais implicações em terapia intensiva. In: Cherregati AL, Amorim CP. Enfermagem em unidade de terapia intensiva. 2. ed. São Paulo: Martinari; 2014. p. 311-26.

14. Morsch CMF. Urgências e emergências em nefrologia. Santos MN, Soares OM. In: Urgência e Emergência na prática de enfermagem. Porto Alegre: Moriá; 2014. p. 1012-21.

15. Bignotto LH, Kallás ME, Djouki RJT, Sassaki MM, Voss GO, Soto CL, et al. Achados eletrocardiográficos em pacientes com doença renal crônica em hemodiálise. J Bras Nefrol [Internet]. 2012 [acesso em 25 nov 2017]; 34(3). Disponível em: http://dx.doi.org/10.5935/0101-2800.20120004.

16. Olesen JB, Gregory YH, Kamper AL, Hommel K, Kober L, Lane DA, et al. Stroke and bleeding in atrial fibrillation with chronic kidney disease. N. Engl. J. Med. [Internet]. 2012 [acesso em 25 nov 2017]; 367(7). 
Disponível em: http://dx.doi.org/10.1056/NEJMoa1105594.

17. Gil CA. Como encaminhar uma pesquisa. 4. ed. São Paulo: Atlas S.A.; 2002. p. 17-21.

18. Silveira DT, Córdova FP. A pesquisa científica. In: Gerhardt TE, Silveira DT. Métodos de pesquisa. Porto Alegre: UFRGS; 2009. p. 33.

19. Newman TB, Browner WS, Cummings SR, Hulley SB. Delineando estudos transversais e de casocontrole. In: Newman TB, Browner WS, Cummings SR, Grady DG, Hulley SB. Delineando a pesquisa clínica: uma abordagem epidemiológica. Porto Alegre: ARTMED; 2008. p. 127-9.

20. Burmeister JE, Mosmann CB, Bau R, Rosito GA. Prevalência de diabetes mellitus em pacientes renais crônicos sob hemodiálise em Porto Alegre, Brasil. J. Bras. Nefrol. [Internet]. 2012 [acesso em 25 nov 2017]; 34(2). Disponível em: http://dx.doi.org/10.1590/S0101-28002012000200003.

21. Sousa AG de, Fichino MZS, Silva GS da, Bastos FCC, Piotto RF. Epidemiology of coronary artery bypass grafting at the Hospital Beneficência Portuguesa. Braz J. Cardiovasc. Surg [Internet]. 2015 [acesso em 05 mar 2019]. 30(1). Disponível em: http://dx.doi.org/10.5935/1678-9741.20140062.

22. Lobo TJ, Pachon CT, Pachon JC, Pachon El, Pachon MZ, Pachon JC, et al. Ablação da fibrilação atrial na disfunção sistólica: evolução clínica e ecocardiográfica. Arq. Bras. Cardiol. [Internet]. 2015 [acesso em 25 nov 2017]; 104(1). Disponível em: http://dx.doi.org/10.5935/abc.20140167.

23. Garcia RJV. Cardioversión eléctrica como modalidade terapêutica em pacientes com fibrilación auricular. Medisan [Internet]. 2018 [acesso em 05 mar 2019]; 22(7). Disponível em: http://scielo.sld.cu/pdf/ san/v22n7/1029-3019-san-22-07-508.pdf.

24. Amorim AMV, Deus RB de, Rodrigues FSM, Malagutti W, Ferraz RRN. Eficácia do citrato como anticoagulante na hemodiálise veno-venosa contínua com ciclo de 60 horas em unidade de terapia intensiva. ConScientiae Saúde [Internet]. 2010 [acesso em 25 nov 2017]; 9(2). Disponível em: http://dx.doi. org/10.5585/conssaude.v9i2.2163.

Recebido: 07/07/2018

Finalizado: 25/04/2019

Autor Correspondente:

Fernando Riegel

Universidade Federal do Rio Grande do Sul

Av. Ipiranga, 3377 - 90610-001 - Porto Alegre, RS, Brasil

E-mail: fernandoriegel85@gmail.com

Contribuição dos autores:

Contribuições substanciais para a concepção ou desenho do estudo; ou a aquisição, análise ou interpretação de dados do estudo - LPP

Elaboração e revisão crítica do conteúdo intelectual do estudo - DSR

Aprovação da versão final do estudo a ser publicado - FR 